Meta

Journal des tradlucteurs

Translators' Journal

\title{
Délivrer la Bible : la théorie d'Eugène Nida
}

\section{Sherry Simon}

Volume 32, numéro 4, décembre 1987

URI : https://id.erudit.org/iderudit/004151ar

DOI : https://doi.org/10.7202/004151ar

Aller au sommaire du numéro

Éditeur(s)

Les Presses de l'Université de Montréal

ISSN

0026-0452 (imprimé)

1492-1421 (numérique)

Découvrir la revue

Citer cet article

Simon, S. (1987). Délivrer la Bible : la théorie d'Eugène Nida. Meta, 32(4),

429-437. https://doi.org/10.7202/004151ar d'utilisation que vous pouvez consulter en ligne.

https://apropos.erudit.org/fr/usagers/politique-dutilisation/ 


\section{DÉLIVRER LA BIBLE : LA THÉORIE D'EUGĖNE NIDA}

Depuis ses origines dans le mythe de l'unanimité miraculeuse des traducteurs de la Septante, la traduction biblique en Occident s'est définie comme une pratique spécifique, différente par ses méthodes et ses exigences de la traduction de textes profanes. Cette spécificité a été réaffirmée tout au long de la tradition, entre autres par SaintJérôme (dans sa célèbre et très influente déclaration sur l'importance de l'ordre des mots dans la traduction biblique) et plus tard par Luther. Avec très peu d'exceptions, les traducteurs bibliques ont travaillé indépendamment des traducteurs de textes profanes1. L'écart entre les deux pratiques, créé par des considérations théologiques, s'est agrandi de siècle en siècle sous le poids toujours plus lourd du savoir accumulé.

Il est donc étonnant que deux des plus éminents théoriciens modernes de la traduction soient des traducteurs bibliques. Et peut-être plus étonnant encore que leurs théories de la traduction soient fondées dans l'expérience et les contraintes de cette activité spécifique ${ }^{2}$. Si la poétique de la traduction proposée par Henri Meschonnic est liée à une réflexion sur la textualité en général, la science de la traduction que vise Nida s'intègre davantage aux approches pragmatiques adoptées par des traductologues contemporains. C'est ainsi que la théorie de Nida, par son accessibilité et par ses visées, s'est vu accorder un rôle important dans les nouvelles institutions de la traductologie.

Doit-on attribuer les divergences profondes entre les deux théories à la très longue histoire religieuse et textuelle qui en fournit l'encadrement, et adopter une attitude relativiste ? Il y a, cependant, dans les divergences entre ces deux penseurs, des enjeux importants pour la théorisation de la traduction. Dans son importante et très incisive critique de la théorie de $\mathrm{Nida}^{3}$, Meschonnic montre que la "science " de Nida en fait cache une idéologie, érigeant en loi un dualisme non théorisé et essentiellement théologique. En construisant sa théorie "générale " de la traduction sur une distinction absolue et non justifiée entre la forme et le sens, Nida laisse sous silence les principaux présupposés théoriques de son projet.

Cette étude se veut une prolongation de la critique de Meschonnic à l'égard de la théorie d'Eugène Nida, en montrant comment l'activité missionnaire à laquelle est destinée la théorie de Nida peut expliquer certains aspects de sa pensée. Nous voudrions nontrer que ce que Nida présente comme une activité neutre, non idéologique, scientifique, est en fait une activité lourde de conséquences sur le plan social et historique.

\section{LES TRADUCTEURS DE LA RÉFORME AU TIERS-MONDE}

Eugène Nida est un pasteur protestant qui a longtempsété associé à l'Institut linguistique d'été, une entreprise de traduction biblique à l'échelle mondiale connue aussi sous le nom de la société Wycliffe ${ }^{4}$. Nida a occupé également le poste de directeur des traductions à l'American Bible Society et travaille également avec l'organisme international United Bible Societies. Depuis trente ans Nida élabore une pensée globale qui place la traduction au centre du processus de communication entre cultures. Des sciences sociales, des théories de la communication et de l'information, mais surtout de la lin- 
guistique moderne, Nida a soustrait les éléments pouvant contribuer à une compréhension systématique de la traduction. Ses très nombreux écrits témoignent toujours d'un grand souci de rigueur intellectuelle ; ils portent aussi la marque de l'exigence pédagogique.

Nida insiste beaucoup sur la nouveauté radicale de sa démarche de traducteur. En fait, tant sur le plan des principes herméneutiques que sur celui de l'impact sociohistorique de son activité, son entreprise ressemble beaucoup à celle des traducteurs protestants de la Réforme.

En opposition aux traditions textuelles catholiques et juives, qui toutes deux voient dans la Bible une épaisseur textuelle, une densité créée par le poids accumulé de l'histoire et du dogme, la Réforme a instauré la notion de la transparence du texte. Sola scriptura mot d'ordre calviniste, déclare l'autonomie des Écritures et le principe de leur autorités. Affranchie de son rapport à une autorité interprétative (le Pape de Rome), la Bible devient un texte accessible à tous, enfin retourné à son " sens premier ", à son sens "littéral ". Malgré les querelles dogmatiques qui ont divisé le Protestantisme en une multitude de sectes différentes, l'idée de l'importance de l'accès immédiat de l'individu à la Bible reste primordiale.

À partir des indications de base que Nida présente en introduction à ses livres (et surtout à son manuel, The Theory and Practice of Translation) on comprend que Nida s'inspire très explicitement des principes de base de la tradition biblique protestante. Des principes herméneutiques énoncés par Luther ${ }^{6}$ Nida retient surtout celui de la transparence de la Bible - l'importance de son sens premier - et le principe de son intelligibilité : "Complete intelligibility should be a matter of life and death for the Bible translator, as it is in the aviation industry" $(1969: 1)^{7}$.

Avec Luther toujours Nida affirme que la Bible n'est pas un document mort mais bien un message vivant qui parle directement au lecteur et agit sur lui. La Bible est l'incarnation d'un acte de communication qui comporte non seulement une fonction informative mais également une fonction expressive et surtout une fonction impérative :

$A$ translator of the Bible must not only provide information which people can understand but must present the message in such a way that people can feel its relevance (the expressive element in communication) and can then respond to it in action (the imperative function). $(1969: 24)$

Cette fonction impérative, la plus importante pour Nida, correspond effectivement à un principe de base de l'interprétation biblique protestante :

Not only is Protestant interpretation grounded in the plenary inspiration of the Scriptures, but it takes also as the counterpart of that truth the great purpose of the Bible, namely to produce a spiritual effect in the life of the man that reads it. The Bible is not an end; it is a means. That is to say that the goal of all interpretation is the spiritual results in the listeners ${ }^{8}$.

De ce principe fondamental découlera une foule de conséquences sur le plan pratique de la traduction. La Bible, non pas un document mais bien un message, largue ses amarres historiques. La Bible doit parler au lecteur d'aujourd'hui comme si Dieu lui parlait maintenant. La qualité atemporelle du message biblique est posée comme principe de base.

Même si Nida reconnaît que les préjugés théologiques du traducteur, les difficultés d'ordre technique, et les interférences culturelles ${ }^{9}$ peuvent venir brouiller cette communication directe, il maintient la transparence comme but idéal.

Tout comme celle des traducteurs de la Réforme, l'entreprise de traduction que dirige Nida a d'importantes conséquences sociales et politiques. Sur le plan de l'impact 
du vaste projet de traduction qu'il a dirigé10, cependant, Nida maintient un silence surprenant. Bien que très conscient de l'importance sociale de la langue, Nida parle peu des effets réels de la traduction de la Bible en des milliers de langues - dont beaucoup auparavant sans écriture. Si la Réforme a eu un impact très important sur le développement des langues vernaculaires et par conséquent des consciences nationales en Europe, l'activité de l'Institut linguistique d'été et les sociétés bibliques ont eu une influence sur la vie des langues qui ont reçu une traduction de la Bible (ou de parties de la Bible) et sur la vie politique. Les traducteurs missionnaires de l'Institut linguistique d'été ont formulé des grammaires et des écritures pour de nombreuses langues de peuples vivant encore en dehors de leurs cultures et économies nationales. Ils se sont penchés même sur des langues parlées seulement par quelques centaines de personnes. La curieuse dialectique qui caractérise l'action de l'Institut dès ses débuts a souvent fait que cette intervention ne serve qu'à mettre en branle le processus d'assimilation aux cultures nationales. L'action de l'Institut continue à avoir des effets sociaux et politiques extrêmement importants au Tiers monde et est étroitement liée à la montée du protestantisme évangélique en Amérique latine.

La grande originalité que revendique Nida ne se situe donc pas sur le plan des principes herméneutiques, comme il semble l'affirmer, mais plutôt dans l'insertion sociale de son activité : la vaste échelle de sa mise en pratique, et la manière dont cette activité est rationalisée. Nida a profondément transformé un domaine traditionnellement attribué aux érudits (et laissé à l'écart des exigences pratiques du quotidien) en lui donnant les mêmes critères d'efficacité qui animent la traduction pragmatique. Voici la traduction biblique devenue une nécessité au même titre que la traduction commerciale, et sa théorie devenue la matière de manuels destinés aux milliers de traducteurs missionnaires à travers le monde.

Là aussi où les principes de Nida se démarquent nettement de ceux de ses prédécesseurs c'est dans son refus de considérer la tradition de la traduction biblique en anglais comme une continuité. La grande lignée de traductions qui a donné naissance à la version King Jámes et ses révisions subséquentes n'est pas respectée ; pour Nida la version King James est son contre-exemple préféré, porteur d'un archaïsme inadmissible. Si Nida refuse de voir son activité en continuité avec les traducteurs de la Réforme qui ont quand même tous insisté sur la nécessité de traduire dans une langue accessible à tous - au moins formulaient-ils ainsi leurs préfaces - il est vrai que la situation de Nida par rapport à la langue est très différente. À l'époque de la Renaissance la traduction constituait un moyen privilégié d'affirmer la valeur des langues vernaculaires. La qualité de la langue d'une traduction revêtait une importance énorme. L'innovation réelle de Nida c'est justement l'abandon de critères stylistiques littérarisants dans la rédaction du texte traduit. On comprend que cette attitude facilite la tâche de traducteurs qui traduisent vers des langues sans tradition littéraire écrite. Elle a aussi présidé à la traduction en langue anglaise intitulée Good News Bible, Today's English Version ${ }^{11}$ où domine un refus total de l'esthétisme. Il faut voir dans cette attitude envers la langue l'indication d'une attitude historique - impensable à d'autres époques.

Ce refus de l'esthétisme est au centre même du projet de Nida. Il se trouve continuellement justifié par Nida dans son opposition entre forme et sens.

\section{LE REFUS DE LA FORME}

Le principal obstacle à la transmission du message transparent et efficace de la Bible, d'après Nida, c'est le trop grand respect de la forme du message original.

Some Christians both national and foreign, tend to adopt a view of the Scripture which is more in keeping with the tenets of Islam than with the Biblical view of revelation, for they re- 
gard the Bible as being essentially a dictated document, rather than one in which the distinct stylistic features and viewpoints of the individual writers are preserved. One must look at the words of the Bible as instruments by which the message is communicated and not as ends in themselves. (1969: 101)

Cet énoncé, autant théologique que linguistique, deviendra la base des recherches de Nida. Empruntant différentes approches linguistiques (la théorie des " noyaux ", l'analyse componentielle du sens) Nida cherchera à montrer que le sens se trouve toujours derrière les mots. Chercher à imiter les structures de surface s'apparente plus à un jeu frivole qu'à une partie de la tâche du traducteur.

Préservant un dualisme rigoureux, Nida marque d'un signe positif tout ce qui se qualifie de " contenu " et englobe sous les notions de " forme" ou de "style " tout ce qui peut être non essentiel au message. Une trop grande attention portée à la forme équivaut pour Nida à une pratique élitiste du langage autant qu'à une compréhension fautive du sens. Dans un modèle textuel qui privilégie la communication efficace du sens, la forme et le style deviennent des éléments qui sont posés comme l'inverse des traits de l'efficacité ${ }^{12}$.

La notion de forme finit par acquérir une grande extension : elle désigne, dans l'origine du texte, les conditions spécifiques de sa formulation dans l'espace et dans le temps. Dans le cas d'un texte qui a eu son origine dans une culture et une histoire lointaine, la forme englobe également les traits du texte qui indiquent cette origine. Le principe de «l'équivalence dynamique » vise donc autant que possible à dégager le message des contraintes formelles qui le lient à une langue, à une culture, à une époque historique particulière. Si le message, au bout de son voyage à travers le temps et l'espace, ne peut pas rester identique dans son apparence extérieure, il peut rester identique par la réponse qu'il suscite.

They (the readers) are not so much concerned with the formal resemblance as with the dynamic equivalence. In other words, they want to be able to interpret the relevance of the message within the context of their own lives, without having to consider or be distracted by the formal structures of the original communicative setting. $(1960: 192)$

Jusqu'où le traducteur peut-il aller dans sa recherche de l'équivalence dynamique ? Nida propose la distinction entre la traduction linguistique (permise) et la traduction culturelle (défendue). Pourtant, il est incapable d'en définir les limites. Nida explique que si la "vigne " peut être remplacée par une plante mieux connue par les populations auxquelles la traduction est destinée, le " figuier » doit être maintenu à cause de sa valeur symbolique et "l'agneau " et la "croix " doivent être maintenus à tout prix même s'il y a risque de difficultés de compréhension (1969: 110). Ici, exégèse, herméneutique et traduction se confondent. Les justifications théoriques de Nida, formulées dans le langage neutre de la scientificité, reviennent à nier tout à fait la notion d'une spécificité textuelle. Le traducteur n'a plus à se sentir lié par les apparences du texte. L'éthique se situe ailleurs : du côté du but du traducteur, du côté des attentes de son public.

\section{TEXTE ET CULTURE : UNE MÊME MISSION}

Le dualisme profond et fondamental que Nida affiche dans sa caractérisation des textes se manifeste également dans sa caractérisation des cultures. Si l'amputation de traits formels opérés par l'équivalence dynamique pose la question de l'identité du texte (quand est-ce que le texte traduit cesse d'être le même que le texte original ?), l'activité du missionnaire face aux cultures qu'il rencontre pose des questions très semblables. Donner le message chrétien à une culture : est-ce changer la culture ou bien la rendre plus proche d'elle-même? Dans l'œuvre de Nida on peut percevoir une continuité très 
nette entre sa caractérisation du texte (le contenu est essentiel et la forme est contingente) et de la culture (les valeurs sont universelles et les coutumes sont contingentes).

Au cours de sa carrière prolifique de théoricien et de vulgarisateur, Nida a écrit plusieurs ouvres anthropologiques qui avaient pour but de mieux préparer le missionnaire à son rôle de communicateur. Ces œuvres définissent pour nous le cadre dans lequel l'activité traductrice de Nida s'insère. Dans Message and Mission, the Communication of the Christian Faith (1960 : 206), Nida écrit :

One of the essential difficulties in determining a theological basis for communication is the necessity of disengaging Christian truth from the cultural forces in which it has been embedded throughout history.

Voici le paradigme central : la vérité chrétienne se situe au-delà des facteurs culturels. Il faut savoir transmettre le message chrétien malgré les cultures. Comment concilier cet énoncé avec la leçon de son livre, Customs and Culture : que le missionnaire doit respecter et surtout comprendre les croyances et pratiques spécifiques des peuples ? S'agit-il de comprendre... pour mieux transformer?

Pour Nida, la compréhension de différentes cultures ne mène pas du tout à un relativisme tolérant. Au contraire : le missionnaire doit savoir distinguer entre les pratiques inoffensives et celles qui transgressent des règles éthiques. (Tout comme il doit pouvoir distinguer entre "Christianity " et "Christendom ".) "The missionary's message must not be formulated as: 'Since this is good for you, it must be true' but rather 'This is true, and therefore it is good'" (1954:255).

Qu'est-ce qui se passe quand le missionnaire (produit d'une culture A) rencontre une culture $B$ en lui apportant le message chrétien $C$ ? Si le missionnaire peut neutraliser l'apport de sa propre culture occidentale, et comprendre la spécificité de la culture vers laquelle il porte son message (qui lui est essentiellement a-culturel, a-historique), le transfert sera effectué sans heurt. Ne seront supprimées que les pratiques manifestement contraires à l'éthique universelle ; la culture restera la " même ". Bien que Nida déclare que sa définition de la culture englobe toute la vie d'une communauté (ses croyances autant que ses pratiques), ses discussions insistent surtout sur la culture comme une collection de coutumes. C'est cette perspective limitée sur la culture qui lui permet d'affirmer que la transmission du message chrétien n'est pas en contradiction avec la continuité culturelle.

Le modèle de ce transfert ressemble beaucoup au modèle à trois niveaux de la traduction proposée par Nida (la culture de la Bible, la culture du traducteur, la culture récipiendaire de la traduction). Le succès des deux activités dépend d'une part de l'effacement de la personne intermédiaire (le traducteur, le missionnaire) et d'autre part de la capacité de celle-ci de distinguer entre l'essentiel et le contingent (la forme et le message, les pratiques et les valeurs universelles). Au centre de ces deux activités de communication est le postulat d'une vérité universelle qui est infiniment " acculturable » puisque au-dessus de toute culture. La vie humaine, comme le texte, est double : d'une part il y a les vérités universelles et d'autre part le nécessaire vêtement linguistique et culturel.

\section{QUELLE SCIENCE DE LA TRADUCTION?}

Que la Bible, le Texte des Textes, ait représenté au cours des siècles des réalités diverses constitue une évidence. Lieu d'une révélation, message urgent, document historique, œuvre littéraire : c'est le même texte qui a donné lieu à toutes ces caractérisations. C'est le sort de tout document, devenu monument culturel et transporté à travers siècles et continents, de recevoir de multiples significations et de jouer des rôles variés. Qu'estce que le texte « universel » sinon le texte qui sait échanger son caractère local contre les 
valeurs atemporelles qu'on croit y déceler. La traduction est souvent la négociation de cet échange.

La traduction sera possible tant qu'il y a reconnaissance d'un fonds de valeurs commun pour permettre l'échange. La traduisibilité, on s'en rend compte, n'est pas une question technique mais bien une décision idéologique. "Shakespeare en langue ourdoue? La Bible dans toutes les langues du monde" (devise de la société Wycliffe) ? Peut-être. Mais il faut ensuite poser la question : jusqu'à quand un texte reste-t-il identique à lui-même dans la traduction ? Peut-on, comme le fait Nida, placer l'identité du côté du récepteur («This is something like market research» (1969:163) et choisir entre les parties essentielles et non essentielles du texte ? Quand la forme constitue-t-elle une responsabilité, une obligation du traducteur?

L'opposition entre forme et fonds a été sacralisée par les études littéraires. De plus en plus, pourtant, on reconnaît que la littérature n'est pas une pratique d'écriture essentiellement différente des autres pratiques mais bien une série de pratiques discursives dont la valeur dépend des conditions de production et de réception ${ }^{13}$. Les théoriciens de la traduction qui, sous l'influence du modèle linguistique, ont refusé d'aborder l'étude de la traduction littéraire ${ }^{14}$ ont opéré une distinction qui ne sert qu'à augmenter le mystère qui entoure la traduction littéraire. Au lieu de poser l'existence de deux types de textes, ceux pour qui la formulation originale est pertinente et ceux pour qui elle ne l'est point, il faut postuler l'existence d'une multiplicité de pratiques discursives — différentes par leurs origines et leurs buts.

La théorie d'Eugène Nida maintient au centre de la théorie de la traduction deux conceptions qui faussent radicalement notre perception de l'activité traduisante. D'une part, sa conception de la forme renforce une distinction absolue entre deux types de textes : le texte littéraire et le texte non littéraire. D'autre part, sa caractérisation du traducteur comme médiateur neutre laisse croire que la traduction est une activité sans composante idéologique. En supposant que le rôle du traducteur peut être celui d'un médiateur tout à fait effacé, Nida néglige tout à fait le rôle social et politique réel du traducteur. Dans le contexte surtout de l'activité des traducteurs de l'Institut linguistique d'été, cet oubli est fondamental. C'est tout le niveau du "rapport à l'Autre "élucidé par Antoine Berman ${ }^{15}$, qui a jusqu'ici été occulté dans la théorie de Nida.

Les limites de la théorie de Nida sont les limites du modèle instrumentaliste de la traduction proposé par la linguistique (voir Kelly $1979: 43$ ). Il escamote totalement la présence du sujet traduisant et de l'histoire dans la traduction. La question de la différence dans la traduction, de l'ouverture de la langue d'arrivée à l'étrangeté du texte de départ, est une question essentiellement historique et idéologique. Voilà le couur du long débat qui anime l'histoire de la traduction, qui oppose l'époque classique française aux Romantiques allemands ${ }^{16}$, qui oppose Eugène Nida à Henri Meschonnic.

Même si le travail récent de Nida fait état d'une plus grande sensibilité aux aspects formels du langage (l'essentiel de ces recherches des dernières années portent en tout cas sur des parties du discours et non pas sur des textes entiers) et même si les traducteurs bibliques inspirés par Nida se montrent aussi beaucoup plus nuancés dans leurs analyses textuelles, la théorie inspirée par Nida n'a pas pour autant adressé la question de la position sociale et du projet du traducteur dans son rapport à l'efficacité de la traduction. Cette affirmation peut sembler paradoxale, si on se rappelle que c'est Nida le premier qui a fermement installé les études de la traduction dans le domaine de la sociolinguistique et qui a fait de la traduction une activité socialement déterminée.

Ce qui est absent, cependant, de la conception sociale de la traduction telle que proposée par Nida c'est la position spécifique du sujet-traducteur face au texte à interpréter et à transmettre. Replacer l'activité traduisante dans l'épaisseur de l'histoire, 
dans les inégalités des rapports sociaux, c'est lui restituer sa dimension proprement politique, les dissymétries de tout ordre qui caractérisent le rapport entre le texte original et le texte traduit. C'est montrer que la pratique des traducteurs et traductrices est nécessairement informée par des valeurs.

Notes

Toutes les références entre parenthèses renvoient aux cuvres de Nida.

1. La spécificité des pratiques est bien attestée dès la Renaissance : Between the translators of the Bible and the translators of other works there were few points of contact... Translators of the Bible were scarcely ever translators of secular works and vice versa. " Flora Ross Amos (1973 (1920)) : Early Theories of Translation, New York, p. 74.

2. Nida déclare de façon très explicite dans sa préface à Towards a Science of Translation (p. ix), que sa théorie générale de la traduction repose sur une matière illustratrice tirée exclusivement du domaine de la traduction biblique. Il espère ainsi construire un pont entre l'activité du traducteur biblique et l'activité traduisante en général.

Henri Meschonnic insiste beaucoup sur le rapport entre sa pratique de traducteur biblique et sa théorie. "La théorie s'est apprise, essayée, autant dans un travail de lecture-écriture que d'écriture ou de traduction-écriture dans les Cinq rouleaux. "(Pour la poétique, p. 13.)

3. Henri Meschonnic (1973) : "D'une linguistique de la traduction à la poétique de la traduction ", Pour la poétique II, Gallimard, pp. 328-366. Une autre critique importante du travail de Eugène Nida se trouve chez Rodney Needhan (Belief, Language and Experience, Univ. of Chicago Press, 1972). Needham montre à quel point le "projet » du traducteur intervient dans la détermination des équivalences (pp. 14-39, 204-205). Eugène Nida est associé au Summer Institute of Linguistics (Institut linguistique d'été) depuis 1937. Avec Kenneth L. Pike, il est reconnu comme le principal théoricien de la traduction de la Bible dans une perspective évangéliste protestante. Nida occupe le poste de directeur des traductions à l'" American Bible Society» et travaille également avec l'organisation internationale "United Bible Societies». Pour une biographie de Nida ainsi qu'une bibliographie de ses écrits, voir : "Eugène $A$. Nida : an Appreciation ", par Eric North, en introduction à : On Language, Culture and Religion : in Honour of Eugène Nida, Mouton, 1974.

4. Il y a dans les débats sur l'autorité des Écritures à l'époque de la Réforme une énonciation très claire de la distinction entre le message de la Bible et sa formulation exacte, entre la Parole et les paroles bibliques. Les implications théologiques de la question sont exposées en grand détail dans : The Authority of Scripture, A study of the Reformation and post-Reformation Understanding of the Bible, J.K.S. Reid (1957) : London.

Malgré cette reconnaissance de l'opacité possible du texte biblique et la pratique de l'interprétation christologique de l'Ancien Testament, Luther lance l'illusion du sens clair et évident de la Bible. David Stacey (1976) : Interpreting the Bible, London, p. 85, cite Luther : Scriptures have a plain, straightforward sense, and that sense and no other provides the true meaning."

5. Pour une exposition des principes herméneutiques de Luther, voir : The Cambridge History of the Bible, vol. II ; Bernard Ramm (1970) : Protestant Biblical Interpretation, Grand Rapids Michigan; et W. Schwarz (1955) : Principles and Problems of Biblical Translation, Some Reformation controversies and their background, Cambridge.

6. Le principe de l'intelligibilité assume une très grande importance dans la théorie de Nida : toute énigme doit avoir sa réponse. Face à l'ambiguïté de l'original, Nida propose un sens conjectural plutôt que la répétition de l'ambiguité. (Voir 1969 : 8 et ailleurs un exemple de " parbar ».) Cette attitude relève du principe de l'autonomie du texte traduit et elle est, d'après Chaim Rabin, particulière à la traduction chrétienne : It is this tendency for the Christian Bible translations to become independent sources of religious authority and emotion which is, to my mind, their most typical trait, and does to the best of my knowledge not exist in other religions with regard to translations of their sacred books" "Cultural Aspects of Bible translations ", Chaim Rabin (1972) : Babel, Vol. 18, № 3, pp. 11-20. 
Si pour Nida les principes de l'intelligibilité et de la transparence vont de soi, ils sont plus problématiques pour certains de ces disciples. Voir par exemple Charles R. Taber (1970) : " Explicit and Implicit Information in Translation ", The Bible Translator, Vol. 21, No 1, January, pp. 1-9; et Jean-Claude Margot (1979) : Traduire ou trahir, Lausanne, Âge d'homme.

8. Bernard Ramm : op. cit., p. 75.

9. Le modèle à trois niveaux que Nida construit pour caractériser le rapport entre les trois cultures impliquées dans le processus de communication traductionnelle (celle de la Bible, celle du traducteur missionnaire, celle du peuple qui recevra la traduction) sert à démontrer que malgré ces brouillages la transmission directe est possible. "Their task (les traducteurs) is to communicate $\boldsymbol{M}_{1}$ in terms of $\boldsymbol{M}_{3}$ with the least possible skewing as the result of $M_{2}$ 》 (1959:18). La traduisibilité de la Bible est augmentée du fait des ressemblances culturelles entre $\mathbf{M}_{1}$ et $\mathbf{M}_{3}$ : "...the so-called Biblical culture exhibits far more similarities with more other cultures than perhaps any other one culture in the history of civilization. This fact makes the Bible so much mare 'translatable' in the many diverse cultures of the world than most books coming out of our own contemporary Western culture» $(1969: 19)$.

10. Pour une analyse des implications culturelles et politiques des activités de l'Institut linguistique d'été, voir : " How to Speak White in Four Thousand Languages", Sherry Simon (1982) : This Magazine, Vol. 16, No 3, July, pp. 33-36. Ou la version française (1982) : "La linguistique au service de la civilisation », le Temps fou, numéro 23, novembre-décembre, pp. 41-44. Voir également : Is God an American ?, Peter Aaby et Soren Hvalkof, London, Survival International and Copenhagen : International Work Group for Indigenous Affairs. Et : Fishers of Men or Founders of Empire? The Wycliffe Bible Translators in Latin America, David Stoll (1983) : London. Dans "Why Translate the Bible into 'New Languages' ? " (The Bible Translator, vol. 23, $\mathrm{n}^{\circ} 4$, October 1972), Nida défend le principe du multilinguisme dans le contexte national des pays du Tiers-monde.

11. Good News Bible, The Bible in Today's English Version, American Bible Society, 1976.

12. Henri Meschonnic : Pour la poétique II, p. 346. Nida ne nie pas l'importance des aspects formels du langage dans certains cas spécifiques comme la traduction de la poésie. La spécificité de la communication poétique résiderait pour Nida justement dans son absence d'efficacité : "For there is a kind of purposeful inexactness in poetry". "Poetry and the Bible Translator ", The Bible Translator, Vol. 33, № 4, p. 348.

13. Voir par exemple Terry Eagleton (1983) : Literary Theory, Oxford. Robert de Beaugrande, dans Factors in a Theory of Poetic Translating (Van Gorcum, Assen, The Netherlands, 1978), souligne l'influence malheureuse de la linguistique américaine dans la séparation de la forme et du contenu (p. 95).

14. Jean Delisle, dans son Analyse du discours : méthode de traduction (Université d'Ottawa, 1981), justifie son refus de traiter des problèmes de la traduction littéraire en invoquant la nature différente-inspirée, universelle de l'œuvre littéraire. Cette sacralisation de l'œuvre littéraire crée un faux écart entre littérature et non littérature, entre traduction littéraire et traduction pragmatique.

15. "Car la traduction n'est pas une simple médiation : c'est un processus où se joue tout notre rapport à l'Autre. " Antoine Berman (1984) : l'Épreuve de l'étranger, Gallimard, p. 287.

16. Berman, l'Épreuve de l'étranger.

\section{REFERENCES}

AMOS, Flora Ross (réd. 1973) : Early Theories of Translation, New York.

BASSNETT-McGUIRE, Susan (1979) : Translation Studies, London, Methuen.

BERMAN, Antoine (1984) : l'Épreuve de l'étranger. Culture et traduction dans l'Allemagne romantique, Paris, Gallimard.

BLACK, SMALLY, Eds. (1974) : On Language, Culture and Religion : in Honour of Eugène Nida, The Hague, Mouton.

BRUCE, F.F. (1978) : History of the Bible in English, New York, Oxford University Press.

de BEAUGRANDE, Robert (1978) : Factors in a Theory of Poetic Translating, Assen/Amsterdam, Van Gorcum.

KELLY, L.G. (1979) : The True Interpreter. A History of Translation Theory and Practice in the West, Oxford.

MARGOT, Jean-Claude (1979): Traduire sans trahir, Lausanne, l'Âge d'homme.

MESCHONNIC, Henri (1973) : Pour la poétique II, Paris, Gallimard. 
MESCHONNIC, Henri (1977) : Numéro spécial : Henri Meschonnic, Esprit, juillet-août.

MESCHONNIC, Henri (1981) : "Traduire la Bible, de Jonas à Jona ", Langue française, no 51.

MESCHONNIC, Henri (1982) : "Le rythme et la traduction ", entretien avec Henri Meschonnic, Esprit, septembre.

NIDA, Eugène A. (1949) : Exploring Animism (avec William Smalley).

NIDA, Eugène A. (1954) : Customs and Culture (Anthropology for Christian Missions), New York, Harpers.

NIDA, Eugène A. (1959) : "The Principles of Translation as Exemplified by Bible Translation ", in Brower, On Translation, New York.

NIDA, Eugène A. (1960) : Message and Mission (The Communication of the Christian Faith), New York, Harpers.

NIDA, Eugène A. (1964) : Towards a Science of Translation, Leiden, Brill.

NIDA, Eugène A. (1969) : The Theory and Practice of Translation (avec Chas. R. Taber), Leiden, Brill. NIDA, Eugène A. (1975) : Exploring Semantic Structures, München, Wilhelm Fink Verlag.

NIDA, Eugène A. (1976) : "Methods of Evaluation of Translation Theories", dans Brislin, Translation. NIDA, Eugène A. (1975) : Componential Analysis of Meaning, La Haye, Mouton.

PARTRIDGE, A.C. (1973) : English Biblical Translation, London.

RABIN, Chaim (1972) : "Cultural Aspects of Biblical Translation ", Babel, Vol. 18, No 3, pp. 11-20.

RAMM, Bernard (1970) : Protestant Biblical Interpretation, Michigan, Grand Rapids.

SCHWARZ, W. (1955) : Principles and Problems of Biblical Translation. Some Reformation Controversies and their Background, Cambridge.

STACEY, Davie (1976) : Interpreting the Bible, London, Sheldon Press. 